\title{
Intranasal Delivery of a Peptide with Antidepressant-Like Effect
}

\author{
Virginia Brown' and Fang Liu*, ${ }^{*, 2}$ \\ 'Department of Neuroscience, Centre for Addiction and Mental Health, Toronto, ON, Canada; ${ }^{2}$ Department of Psychiatry, University of Toronto, \\ Toronto, ON, Canada
}

\begin{abstract}
A critical issue in drug development is developing effective, noninvasive delivery routes to the central nervous system (CNS). Major depressive disorder (MDD) is an illness associated with significant morbidity. Even with multiple antidepressant trials, I0- I5\% of patients continue to experience persistent depressive symptoms. We previously developed an interfering peptide that has antidepressant-like effects in rats when injected directly into the brain. To be clinically viable, it must demonstrate efficacy via a noninvasive administration route. We report here that the interfering peptide designed to disrupt the interaction between the DI and D2 dopamine receptors can be delivered to relevant brain areas using the Pressurized Olfactory Device (POD), a novel intranasal delivery system developed by Impel NeuroPharma. We validate this delivery method by demonstrating that, at doses $\geqslant 1.67 \mathrm{nmol} / \mathrm{g}$, the DI-D2 interfering peptide has a significant antidepressant-like effect comparable to that of imipramine in the forced swimming test (FST), a common test for antidepressant efficacy. The antidepressant-like effect of the interfering peptide can be detected for $2 \mathrm{~h}$ after intranasal administration. Furthermore, we show that the interfering peptide disrupts the DI-D2 interaction and it can be detected in the prefrontal cortex after intranasal administration. This study provides strong preclinical support for intranasal administration of the D I-D2 interfering peptide as a new treatment option for patients suffering from MDD.

Neuropsychopharmacology (20I4) 39, 2 I3I-2I4I; doi:I0.1038/npp.20I4.6I; published online 23 April 2014
\end{abstract}

\section{INTRODUCTION}

The neurotransmitter dopamine is involved in many processes within the brain, including motor control, cognition, reward, emotion, and pleasure. Dopamine exerts its effects through five unique dopamine receptors, termed D1 through D5. These receptors are G protein-coupled receptors (GPCRs) that contain seven transmembrane domains and initiate intracellular signaling cascades (Beaulieu and Gainetdinov, 2011). In addition to existing as unique receptors, dopamine receptors can also couple with other proteins and receptors to form functional heterodimers that activate signaling cascades, independent from those activated by each component receptor (Maggio et al, 2009; Missale et al, 2010). Dopamine receptors also form heterodimers through direct protein-protein interactions between D1 and D2 receptors (Free et al, 2007; Pei et al, 2010; Perreault et al, 2010; So et al, 2009), D1 and D3 receptors in the striatum (Ridray et al, 1998; Schwartz et al, 1998), D2 and D5 receptors (O'Dowd et al, 2013; So et al, 2009), D1 receptors and NMDA receptors (NMDARs) (Lee

\footnotetext{
*Correspondence: Dr F Liu, Department of Neuroscience, Centre for Addiction and Mental Health, 250 College Street, Toronto, ON M5T IR8, Canada, Tel: + | 416979 4659, Fax: + | 416979 4663, E-mail: f.liu.a@utoronto.ca

Received 21 January 2014; revised 25 February 2014; accepted 10 March 2014; accepted article preview online 17 March 2014
}

and Liu, 2004; Pei et al, 2004), and D5 and GABA-A receptors (Liu et al, 2000), among other transmembrane and cytoplasmic proteins. Dopamine D1 and D2 receptors couple in this manner and the functional heterodimer may play a role in the pathogenesis of major depressive disorder (MDD) (Pei et al, 2010; Wong and Liu, 2012).

MDD is a common, serious psychiatric condition that accounts for $4.4 \%$ of total global disease burden (Mann, 2005) and is often left undiagnosed and untreated in patients (Cuijpers, 2011; Mojtabai, 2009; van Beljouw et al, 2010). Furthermore, many patients do not respond to available pharmacological or psychological treatment for MDD, with $>50 \%$ of patients not responding to first-line pharmacological treatment (Papakostas, 2009; Thase et al, 2007). Pei et al (2010) demonstrated that the D1-D2 heterodimer is upregulated in the striatum of patients with MDD. After identifying a 15 -amino acid sequence within the third intracellular loop of the D2 long receptor isoform, Pei et al (2010) designed a 15-amino acid peptide that is able to disrupt this interaction both in vivo and in vitro. Furthermore, the D1-D2 interfering peptide has an antidepressant effect in the forced swimming test (FST) and the learned helplessness (LH) task, two preclinical tests for antidepressant efficacy (Pei et al, 2010). As the invasive administration methods used limited the clinical validity of this study, we investigated whether the D1-D2 interfering peptide could be delivered to the PFC in a less-invasive, clinically applicable manner. 
Many protein and peptide drugs do not cross the blood brain barrier (BBB) in effective concentrations. Direct injection into the brain or cerebral spinal fluid may lead to effectiveness but is extremely invasive and not practical in the clinical setting. Intranasal (IN) drug delivery offers a noninvasive alternative for getting protein and peptide drugs into the brain by utilizing the olfactory neuronal distribution pathways in the cribriform plate that lead to direct nose-to-brain drug distribution (Dhuria et al, 2010). IN administration can lead to a direct transfer of protein and other types of drugs from the nose to the brain in similar or higher concentrations than can be obtained by systemic administration (Hoekman and Ho, 2011a; Shemesh et al, 2012; Yang et al, 2013). In some cases, IN drug delivery can result in 100 -fold increases in protein drug concentration in multiple brain regions (Dhuria et al, 2010). A number of proteins including insulin and nerve growth factor have been delivered effectively to the central nervous system (CNS) IN in both animals and humans (De Rosa et al, 2005; Renner et al, 2012; Shemesh et al, 2012). Some of the other advantages of IN drug delivery include the possibility for at-home use, multiple dosings a day, and its cost effectiveness.

Depositing drug on the difficult to target olfactory region of the nasal cavity is critical to achieving direct nose-tobrain delivery. Impel NeuroPharma (Seattle, Washington) has developed a Pressurized Olfactory Delivery (POD) device to specifically deposit a majority of drug on the olfactory region of the nasal cavity, as illustrated in Figure 1a (Hoekman and Ho, 2011a, b). Impel has created POD devices for rats, primates, and humans to ensure easy scale-up from preclinical to clinical studies. Administering our interfering peptide with the POD device has the potential to accelerate the development of this therapeutic and allow rapid adoption as a treatment for depression.

The purpose of this study is to test whether we can effectively administer the D1-D2 interfering peptide to the brain using IN delivery. Intranasal delivery is clinically applicable, offers a direct pathway to the brain, and is a noninvasive method to target therapeutics to the CNS (Dhuria et al, 2010; Jansson and Bjork, 2002; Ross et al, 2008). We show that at sufficient doses, IN administration of the D1-D2 interfering peptide has a significant antidepressant effect in the FST for up to $2 \mathrm{~h}$ after IN administration. Furthermore, the interaction between D1R and $\mathrm{D} 2 \mathrm{R}$ is reduced in animals that received IN injections of the D1-D2 interfering peptide, suggesting that the antidepressant efficacy of this peptide is because of its ability to disrupt the interaction between D1R and D2R.

\section{MATERIALS AND METHODS}

\section{Animals}

Adult male Sprague-Dawley rats (Charles River Laboratories, Wilmington, MA) were used in all experiments. Rats were pair-housed at a constant temperature $\left(20-23{ }^{\circ} \mathrm{C}\right)$ on a 12-h light/dark cycle with unrestricted food and water. After arriving at the facility, rats were acclimatized for 1 week before behavioral testing and injections began. All rats weighed 300-350 g when they underwent behavioral testing. All experimental procedures were approved by the animal care committee at the Centre for Addiction and Mental Health (Toronto, ON).

\section{IN and IP Injection Procedures}

Animals were anesthetized using 5\% isoflurane (Benson Medical Industries) for $3 \mathrm{~min}$. Rats were then placed in a supine position and dosed with the POD developed by Impel NeuroPharma. The POD tip (with the relevant dose) was inserted $\sim 8-10 \mathrm{~mm}$ into the rat's nostril, angled toward the olfactory epithelium (toward the top of the head) and the propellant can was fired for $1 \mathrm{~s}$ (Figure 1a). After $2 \mathrm{~s}$, the POD tip was slowly removed. Animals were replaced in the anesthetic chamber in the supine position for $4 \mathrm{~min}$ at decreasing isoflurane concentrations, and then replaced in their cages. Rats that bled from their nostrils after IN administration were eliminated from the study. The time of injection was recorded as the time at which the animals woke up from the anesthesia. Animals given intraperitonal (IP) injections were also anesthetized following the same procedure outlined above, but were given IP injections into the abdominal cavity and not IN injections.

\section{Substances Injected IN and IP}

In each experiment, animals received the same total number of IN injections (3-4) in alternating nostrils over a 24-h period. Animals received filtered saline $(0.9 \% \mathrm{NaCl}$, 8-12 $\mu \mathrm{l}$ ), the D1-D2 interfering peptide (TAT-D1-D2-IPep; $8-12 \mu \mathrm{l}, 50 \mathrm{mM})$, or a amino acid membrane-permeable TAT peptide (TAT-Pep; $8-12 \mu \mathrm{l}, 50 \mathrm{mM}$ ) from the human immunodeficiency virus 1 (HIV1) TAT protein (Schwarze et al, 1999). The 9-amino acid TAT peptide sequenced from the HIV1 TAT protein (YGRKKQRRR) (Schwarze et al, 1999) rendered the peptides cell permeable. Peptides were synthesized by Gen Script (New Jersey, USA) and/or Biomatik (Cambridge, Ontario). Imipramine hydrochloride (15 mg/ml, Sigma-Aldrich) was given at a dose of $15 \mathrm{mg} / \mathrm{kg}$ via IP injections.

\section{FST Procedure and Data Analysis}

The FST is an acute test for antidepressant efficacy developed by Porsolt et al (1977). On the first day of the test, animals undergo a training session where they are placed in an inescapable plexiglass cylinder $(60 \mathrm{~cm}$ high and $20 \mathrm{~cm}$ in diameter) filled with $40 \mathrm{~cm}$ of water at a temperature of $25 \pm 0.2^{\circ} \mathrm{C}$ for a 15 -min training session on day 1 of the test. The water was changed between each testing session. In accordance with the dosing schedule established in the literature and used previously (Lucki, 1997; Pei et al, 2010; Porsolt et al, 1978; Porsolt et al, 1977) animals were dosed three times between the training and testing sessions: $30 \mathrm{~min}$ after the 15 -min training session, $5 \mathrm{~h}$ after training, and $1 \mathrm{~h}$ before undergoing the FST. At $24 \mathrm{~h}$ after the training session and $1 \mathrm{~h}$ after the last behavioral intervention, the rat was replaced in the same cylinder for $5 \mathrm{~min}$. After both the training and test FST sessions, rats were towel-dried and placed in a heated cage for a minimum of $15 \mathrm{~min}$.

The 5-min session of the FST was video recorded and animal behavior during the FST was scored at a later date 


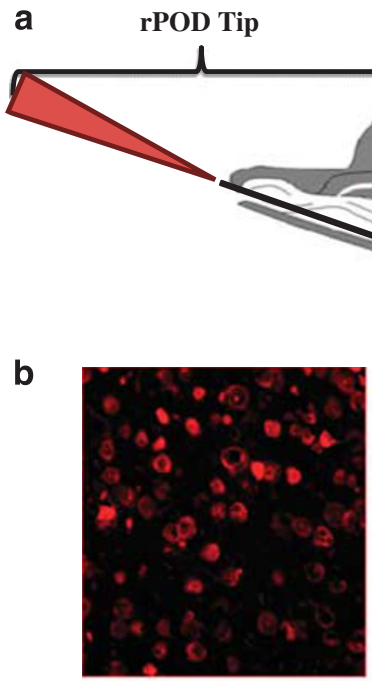

Target Dosing Region
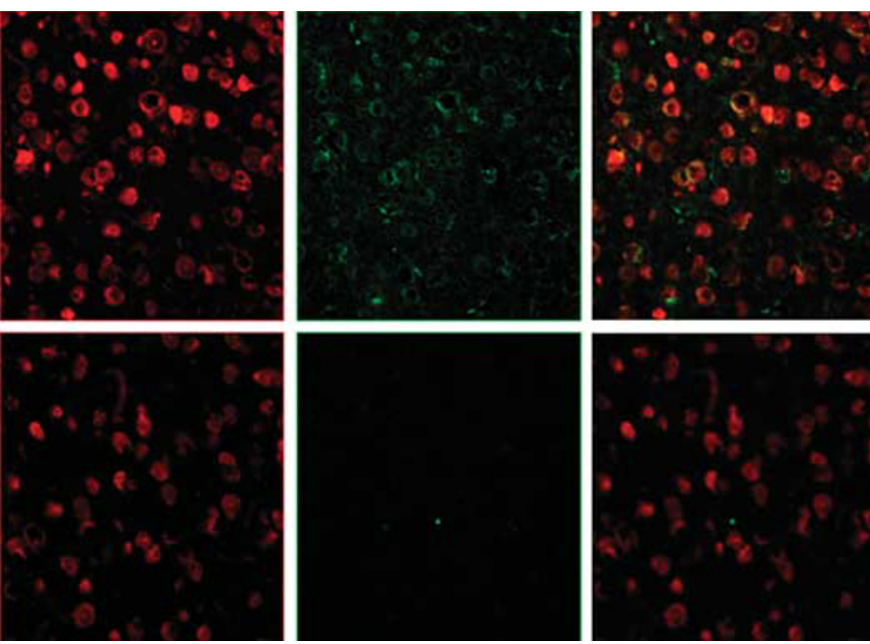

TAT-D1-D2-

FLAG-IPep
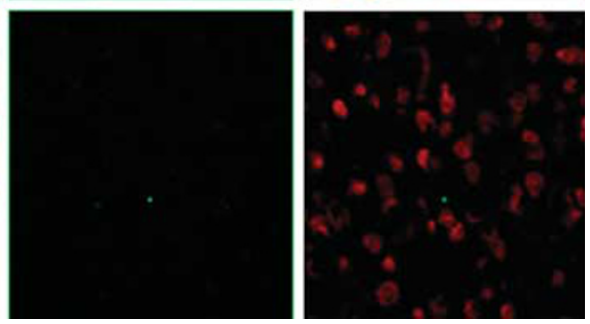

C

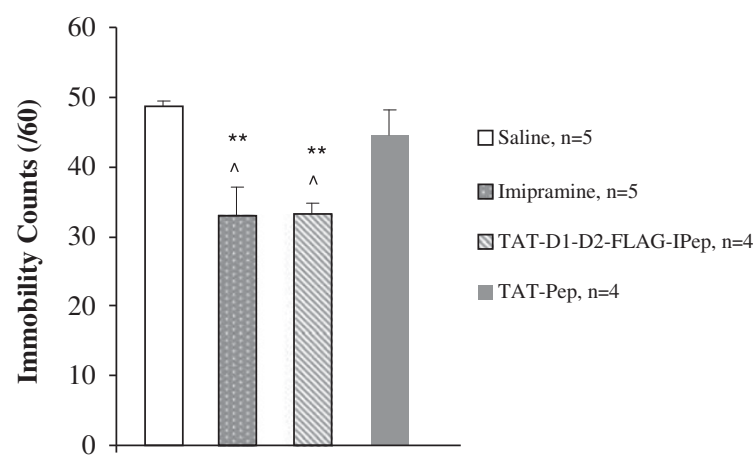

Figure I A FLAG-tagged D I-D2 interfering peptide has an antidepressant effect after intranasal administration. (a) The rPOD working model: the rPOD tip is designed to navigate the complex naris of the rat while targeting the olfactory epithelium. The rPOD is positioned deep into the nasal cavity, where its close proximity with the target-dosing region gives optimal deposition for nose-to-brain delivery. (b) Immunofluorescent staining of PFC slices from animals that received intranasal injections of the DI-D2-FLAG-tagged interfering peptide $(1.67 \mathrm{nmol} / \mathrm{g})$ or saline. Prefrontal cortical slices were stained with antiFLAG antibodies and imaged at $\times 25$ using a confocal microscope. Panels show Neurotrace 530/6 I5 (left), Cy2-conjugated secondary antibodies to antiFLAG (middle), and merged (right) channels. (c) The DI-D2-FLAG interfering peptide has an anti-immobility effect in the FST after intranasal administration. TAT-DI-D2-FLAG-IPep and TAT-Pep administered intranasally at a dose of $1.67 \mathrm{nmol} / \mathrm{g}$. Data were analyzed using one-way independent groups ANOVA followed by Newman-Keuls post hoc multiple comparisons tests. ${ }^{*} * \mathbf{p}<0.01$ compared with saline; ${ }^{\wedge} p<0.05$ compared with TAT-Pep. No significant difference was observed between behavior in animals who received saline or TAT-Pep. Error bars represent SEM.

after the experimenter was blinded to the treatment groups. The animal's activity during the FST was segregated into four behaviors, in keeping with the literature and previous studies (Lucki, 1997; Pei et al, 2010; Porsolt et al, 1977): immobility, swimming, climbing, and diving. Mean behavior counts across all treatment groups were analyzed by one-way independent groups analysis of variance (ANOVA). The post hoc Newman-Keuls multiple comparisons tests were used to evaluate differences across individual groups, as necessary.
To evaluate the efficacy of the D1-D2 interfering peptide at various doses, we compared immobility behavior in animals that received IN injections of $0.5 \mathrm{nmol} / \mathrm{g}(n=6)$, $1 \mathrm{nmol} / \mathrm{g}(n=6), 1.67 \mathrm{nmol} / \mathrm{g}(n=7), 2 \mathrm{nmol} / \mathrm{g}(n=8)$, and $4 \mathrm{nmol} / \mathrm{g}(n=3)$ TAT-D1-D2-IPep to those who received IN injections of saline ( $n=16$ across all doses), TAT-Pep $(1.67 \mathrm{nmol} / \mathrm{g}, \quad n=6)$, or imipramine $(15 \mathrm{mg} / \mathrm{kg}, \quad n=17$ across all doses).

To evaluate the length of time the D1-D2 interfering peptide remains active in the body, we varied the amount of 
time between IN injection of the TAT-D1-D2-IPep or TATPep and the 5-min FST. We compared immobility behavior in animals that received IN injections of the D1-D2 interfering peptide or TAT-Pep $(1.67 \mathrm{nmol} / \mathrm{g})$ at $1,2,3$, or $4 \mathrm{~h}(n=5-6$ per treatment and time point) before the FST with those who received IN injections of saline $(n=6)$ or IP injections of imipramine $(15 \mathrm{mg} / \mathrm{kg}, n=6)$ at 1 hour before the FST. In each of these experiments, immobility behavior across treatment groups at each time point was compared using one-way independent groups ANOVA followed by Newman-Keuls multiple comparisons tests.

\section{Locomotor Activity Test}

We tested whether the D1-D2 interfering peptide had an effect on activity during a 30-min locomotor activity test. Rats that had previously been exposed to the FST were given TAT-D1-D2-IPep (IN, $2.0 \mathrm{nmol} / \mathrm{g}, n=5$ ), saline (IN, $n=5$ ), TAT-Pep (IN $2.0 \mathrm{nmol} / \mathrm{g}, n=5$ ), or $15 \mathrm{mg} / \mathrm{kg}$ imipramine (IP, $n=5$ ) three times before the open field test: at $24 \mathrm{~h}$ before the test, $19 \mathrm{~h}$ before the test, and $1 \mathrm{~h}$ before testing. Animals used in this experiment were kept in their original FST treatment groups.

To record locomotor activity, rats were placed in a custom-made locomotor activity recording apparatus. Each animal was placed in a $20 \mathrm{~cm}$ high, $20 \mathrm{~cm}$ wide, and $30 \mathrm{~cm}$ long cage (standard housing cage) in the locomotor apparatus for $30 \mathrm{~min}$ in a dark room. Rats had not previously been exposed to the testing room or to the locomotor activity boxes. An array of 11 infrared photocells was placed along the long axis of the cages. Interruption of infrared beams (Beam Breaks) was used as a measure of locomotor activity. Locomotor activity was recorded in 5-min bins and for the entire $30 \mathrm{~min}$. Total activity data were analyzed via one-way independent groups ANOVA, followed by Newman-Keuls multiple comparisons tests, using Prism Software (GraphPad Software). The locomotor activity at various time points during the test was analyzed by two-way independent factors ANOVA with treatment group (saline, imipramine, TAT-Pep, and TAT-D1-D2-IPep) and time point $(5,10,15,20,25$, and $30 \mathrm{~min})$ as main factors.

\section{Co-Immunoprecipitation}

Animals were killed the day they completed the FST or, if applicable, the open field test. Animals were anesthetized for $3 \mathrm{~min}$ with $5 \%$ isoflurane and were decapitated. Their brains were quickly removed and relevant tissue areas were dissected on ice and stored at $-80^{\circ} \mathrm{C}$ for subsequent use in biochemical assays. We investigated the co-immunoprecipitation of the D1 receptor by an antibody against the D2 receptor in the PFC of animals who had received IN injections of the D1-D2 interfering peptide or saline. We compared animals who had been given IN injections of TAT-D1-D2-IPep (IN, $1.67 \mathrm{nmol} / \mathrm{g}, n=3$ ) or saline (IN, $n=3$ ) and had been exposed to the FST. For coimmunoprecipitation, solubilized proteins from the PFC and striatum $(500 \mu \mathrm{g})$ from each animal were incubated with $1 \mu \mathrm{g}$ goat polyclonal anti-D2DR (N-19, Santa Cruz Biotechnology) and protein A/G PLUS agarose beads (Santa Cruz Biotechnologies) overnight. A control sample was incubated with polyclonal Goat IgG (Sigma-Aldrich) to confirm the absence of nonspecific immunoprecipitation.

After incubation, the immunoprecipitated proteins were washed and incubated with SDS sample buffer (Bio-Rad) at $37^{\circ} \mathrm{C}$ for $40 \mathrm{~min}$ before being separated from the Protein A/ G PLUS-agarose beads using centrifugation. Immunoprecipitated proteins were then subjected to separation using $10 \%$ SDS-Page gels, transferred onto nitrocellulose membranes, and immunoblotted overnight using anti-D1DR (D187, Sigma-Aldrich). Each immunoblot included samples from saline and TAT-D1-D2-IPep treatment groups, a control sample incubated with goat IgG (Sigma-Aldrich), and $75 \mu \mathrm{g}$ of tissue-extracted input protein from PFC tissue. After overnight incubation, secondary antibodies conjugated with horseradish peroxidase were applied to the blots for $\sim 2 \mathrm{~h}$. After washing, immunoblots were developed with ECL reagent (GE Healthcare) and imaged using a Bio-Rad ChemiDoc MP system (Bio-Rad Technologies). To quantify the expression of protein, we conducted densitometry analyses using ImageLab software (Bio-Rad Technologies). Densitometry data were analyzed using two-tailed, unpaired Student's $t$-tests (Prism Software, GraphPad). Co-immunoprecipitations for each sample were repeated a minimum of three times.

\section{Immunofluorescence and Confocal Microscopy}

To visualize the peptide in the prefrontal cortex after IN administration, an 8-amino acid FLAG-tag (Sequence: DYKDDDDK) (Hopp et al, 1988) was fused to the C-terminal of the interfering peptide. After IN administration of TAT-D1-D2-FLAG-IPep or saline and behavioral testing, animals were killed by transcardiac perfusion followed by perfusion with $60 \mathrm{ml} 4 \%$ paraformaldehyde (in PBS). Subsequently, whole brains were dissected and stored in $4 \%$ paraformaldehyde overnight. The next day, brains were transferred to a $20 \%$ sucrose cryoprotection solution for $48 \mathrm{~h}$. The tissue was then stored at $-80^{\circ} \mathrm{C}$ for subsequent use.

The olfactory bulbs and prefrontal cortex of rat brains from each condition (TAT-D1-D2-FLAG-IPep and Saline) were cut into $12 \mu \mathrm{M}$ sections using a cryostat. Brain slices were blocked for nonspecific antibody interactions using $5 \%$ donkey serum (Sigma-Aldrich) for $1 \mathrm{~h}$, before staining overnight with an anti-FLAG monoclonal antibody (Mouse monoclonal, M2, Sigma-Aldrich). Sections were then incubated with a secondary immunofluorescent antibody (Donkey anti-Mouse Cy2-conjugated antibody, Jackson Immuno Research Laboratories) before being counterstained with NeuroTrace 530/615 red fluorescent Nissl Stain (Molecular Probes, Invitrogen). Sections were mounted on slides with PureGold Anti-Flag Mounting Reagent (Molecular Probes, Invitrogen) and stored at $4{ }^{\circ} \mathrm{C}$. Sections were visualized and imaged using a Zeiss LSM 510 confocal microscope. Images of PFC brain slices in both conditions were taken under $\times 25$ magnification. Cy2 Immunofluorescence was imaged using an Argon laser with maximum excitation at $488 \mathrm{~nm}$. To detect the NeuroTrace 530/615 stain, we used a Helium 1 laser with maximum excitation at $530 \mathrm{~nm}$. Images were overlayed using Image J software. 


\section{RESULTS}

A FLAG-Tagged D1-D2 Interfering Peptide Can be Detected in the PFC after IN Administration and Has an Anti-Immobility Effect in the FST

As an initiation step to test whether the D1-D2 interfering peptide is detectable in the PFC after IN delivery, we administered a D1-D2 interfering peptide with an additional 8-amino acid FLAG tag using the POD and subsequently visualized it in the PFC using immunofluorescent microscopy. After completing the FST test, PFC slices from rats in the D1-D2-FLAG interfering peptide and the saline treatment groups were stained using an anti-flag immunofluorescent antibody. As shown in Figure 1b, we were able to detect the presence of the D1-D2 interfering peptide, whereas slices from the saline treatment group had little to no immunofluorescence. The FLAG-tagged D1-D2 interfering peptide could be detected both intracellularly in neuronal cell bodies within the medial and lateral PFC and extracellularly, implying that the interfering peptide can be delivered to PFC via IN delivery.

We then compared immobility behavior after IN administration of the FLAG-tagged D1-D2 interfering peptide in FST. TAT-peptide, saline, and imipramine were used as control groups. As shown in Figure 1c, the FLAG-tagged D1-D2 interfering peptide significantly decreased immobility behavior during the FST to an extent comparable with imipramine and significantly different from that of saline or TAT-peptide $(\mathrm{F}(3,14)=7.746, p<0.05, n=4-5$ per group).

\section{The D1-D2 Interfering Peptide Has an Antidepressant} Effect in the FST at Doses of $>1.67 \mathrm{nmol} / \mathrm{g}(5.75 \mathrm{mg} / \mathrm{kg})$

To further confirm the behavioral effect of the D1-D2 interfering peptide and to explore the efficiency of IN delivery, we tested the antidepressant-like effect of the D1D2 interfering peptide at various concentrations. We chose the initial dose of $1.67 \mathrm{nmol} / \mathrm{g}(5.75 \mathrm{mg} / \mathrm{kg})$ body weight based on the fact that previous studies suggested that the efficiency of IN delivery to CNS is $1-5 \%$ of the total IN dose (Dhuria et al, 2010). Our previous study indicated that the direct microinjection dose required for the D1-D2 interfering peptide to have an antidepressant-like effect is $5 \mathrm{nmol}$ (Pei et al, 2010). For a rat weighing $300-325 \mathrm{~g}$, we calculated that a sufficient IN dose would be $\sim 500 \mathrm{nmol}(5 \mathrm{nmol} \times$ $100)$. As $\mathrm{nmol} / \mathrm{g}$ body weight, the dose is $1.67 \mathrm{nmol} / \mathrm{g}$ $(500 \mathrm{nmol} \div 300 \mathrm{~g} ; 5.75 \mathrm{mg} / \mathrm{kg}$ ).

Animals that received IN administration of the D1-D2 interfering peptide at $1.67 \mathrm{nmol} / \mathrm{g}$ were significantly less immobile compared with those that received saline or the control TAT peptide (Figure 2a). Furthermore, the behavior of animals that received the D1-D2 interfering peptide was comparable to those that received imipramine. A one-way independent groups ANOVA with treatment group as the main factor revealed that the D1-D2 interfering peptide statistically decreased the observed immobility behavior when compared with rats that received IN injections of saline or TAT-peptide (Figure 2a).

We then extended the IN dose of the peptide to $0.5,1,2$, and $4 \mathrm{nmol} / \mathrm{g}(1.72,3.44,6.86,13.77 \mathrm{mg} / \mathrm{kg}$, respectively). As shown in Figure $2 \mathrm{~b}$, the D1-D2 interfering peptide had a
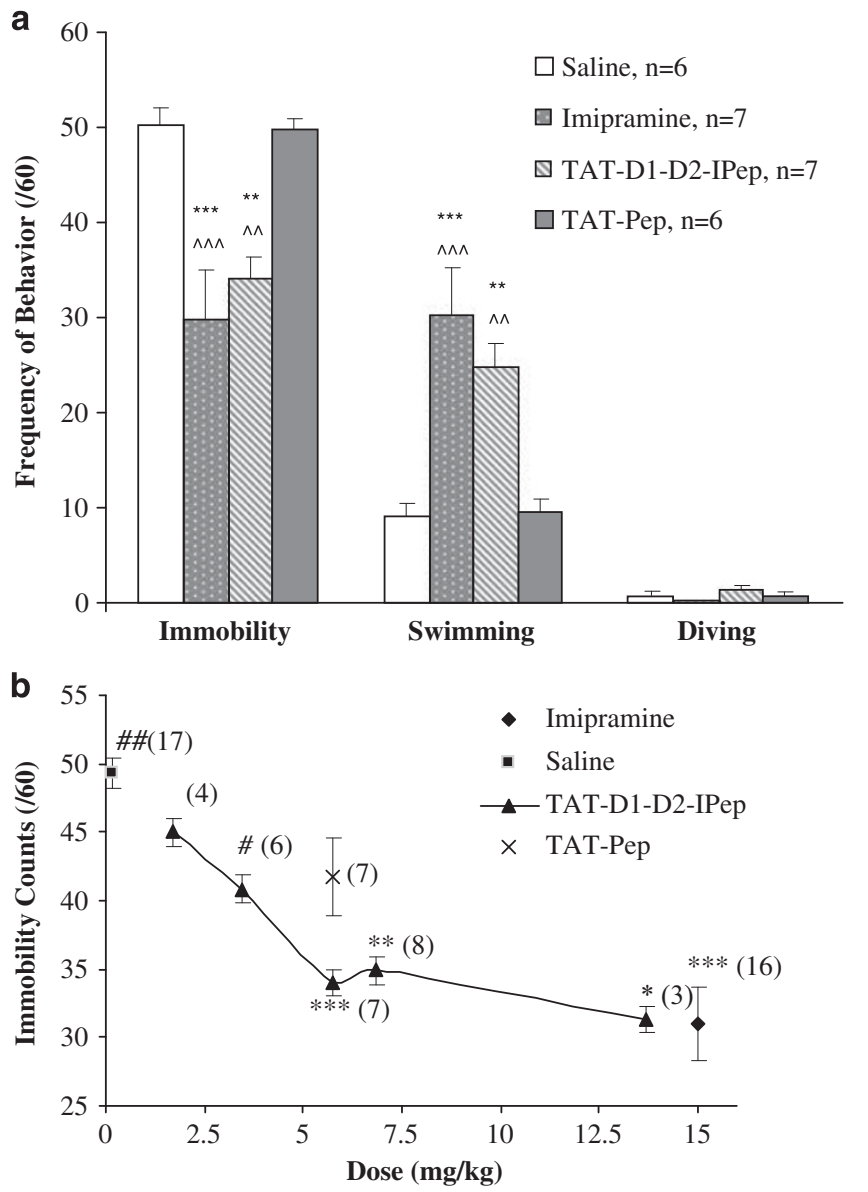

Figure 2 The DI-D2 interfering peptide has an antidepressant effect in the FST when administered intranasally. (a) Effect of the DI-D2 interfering peptide on immobility, swimming, and diving behaviors during the 5-min FST. After intranasal administration using the POD, the DI-D2 interfering peptide (dose $=1.67 \mathrm{nmol} / \mathrm{g}$ ) significantly decreases immobility and increases swimming behavior in the FST. Data for each standard behavior (immobility, swimming, and diving) were analyzed using one-way independent groups ANOVA followed by Newman-Keuls post hoc multiple comparisons tests. $* * * 0.01$, $* * * * p<0.0$ I compared with saline; $\wedge \wedge p<0.01, \wedge \wedge \wedge p<0.00$ I compared with TAT-Pep. No significant difference was observed between behavior in animals who received TAT-DI-D2-Ipep or imipramine. (b) Effect of various doses of the DI-D2 interfering peptide on immobility behavior during the 5-min FST. Data at each dose were compared using one-way independent groups ANOVA. ${ }^{*} * * P<0.001$, ${ }^{* *} p<0.01$, ${ }^{*} p<0.01$ compared with saline; ${ }^{\# \#} p<0.0$ I, ${ }^{\#} p<0.05$ compared with imipramine. Numbers below data points are number of animals per group. Error bars represent SEM.

similar anti-immobility effect at IN doses of $\geqslant 1.67 \mathrm{nmol} / \mathrm{g}$ $(5.75 \mathrm{mg} / \mathrm{kg})$ : dose $4.0 \mathrm{nmol} / \mathrm{g}(\mathrm{F}(2,6=9.207, p<0.01)$ and dose $2.0 \mathrm{nmol} / \mathrm{g} \quad(\mathrm{F}(3,27)=6.836, p<0.01)$. The D1-D2 interfering peptide did not have a significant anti-immobility effect at doses <1.67nmol/g, dose $1.67 \mathrm{nmol} / \mathrm{g}$ $(\mathrm{F}(3,21)=12.25, \quad p<0.001), \quad$ dose $1.0 \mathrm{nmol} / \mathrm{g} \quad(\mathrm{F}(2,19)$ $=9.653, p<0.01)$, and dose $0.5 \mathrm{nmol} / \mathrm{g}(\mathrm{F}(3,20)=7.37$, $p<0.01)$. There was no statistically significant difference between immobility behavior in groups that received saline or TAT-peptide (all doses, $p>0.05$ ), whereas animals that received imipramine $(15 \mathrm{mg} / \mathrm{kg})$ consistently demonstrated a significant decrease in immobility behaviors comparable to those that received saline $(p<0.05)$ and TAT-peptide (all TAT-peptide doses, $p<0.05$; Figure $2 \mathrm{~b}$ ). 
The D1-D2 Interfering Peptide Has an Antidepressant Effect in the FST for $2 \mathrm{~h}$ after IN Administration

Next, we determined the duration of the behavioral antidepressant effect of the D1-D2 interfering peptide. To do this, we increased the length of time between the final IN injection and the 5-min FST, while keeping the total amount of IN injection treatments and the doses that animals received constant. We compared animals that received IN injections of the D1-D2 interfering peptide at $1,2,3$, and $4 \mathrm{~h}$ before the 5-min FST to those that received TAT-peptide, saline, and imipramine. We treated the imipramine and saline treatment groups as positive and negative controls, respectively, and administered these treatments $1 \mathrm{~h}$ before the FST.

At each time point, we compared immobility behavior during the 5-min FST in animals that had received the D1D2 interfering peptide with those that received the TATpeptide, saline, or imipramine using one-way independent groups ANOVA $(2 \mathrm{~h}: \mathrm{F}(3,19)=5.399, p<0.01, n=5-6$ per group; $3 \mathrm{~h}: \mathrm{F}(3,20)=4.669, p<0.01, n=5-6$ per group; and $4 \mathrm{~h}: \mathrm{F}(3,19)=3.727, p<0.05, n=5-6$ per group). At $2 \mathrm{~h}$ after IN administration, animals that received IN administration of the D1-D2 interfering peptide displayed significantly decreased immobility compared with animals that received TAT-peptide $(p<0.05)$ or saline $(p<0.05)$ and had comparable immobility behavior to animals treated with imipramine $(p>0.05)$. However, this effect was no longer statistically significant at the 3 and $4 \mathrm{~h}$ time points, where groups that received the D1-D2 interfering peptide no longer demonstrated significantly different immobility behaviors from those that received saline or TAT-pep ( $p>0.05$, both groups; Figure 3).

\section{IN Administration of the D1-D2 Interfering Peptide Disrupts the Interaction between D1R and D2R in the PFC}

The D1-D2 interfering peptide is designed to specifically disrupt the interaction between the C-terminal cytosolic tail of the D1 receptor and the third intracellular loop of the D2-long receptor. Our laboratory previously demonstrated that it significantly disrupts this interaction in vivo, after administration via direct microinjections to the PFC and other brain areas (Pei et al, 2010). Here, we investigated whether IN administration of the D1-D2 interfering peptide was also able to disrupt the protein-protein interaction between the D1 and D2 receptors. We compared the co-immunoprecipitation of the D1 receptor by an anti-D2 antibody in tissue from the PFC of animals that had received IN injections of the D1-D2 interfering peptide or saline and had been exposed to the FST (Figure 4a). Quantification of the immunoblots revealed that the immunoprecipitation of the D1 receptor by anti-D2 receptor was significantly decreased in animals that received the D1-D2 interfering peptide compared with those in the saline treatment groups $(n=3$ per group; $\mathrm{t}(4)=3.872$; $p<0.05$; Figure $4 \mathrm{~b}$ ). Total protein levels of the D1 and the D2 receptor were unaffected by IN administration of the D1-D2 interfering peptide (Figure $4 \mathrm{c}$ and $\mathrm{d}$ ). This result suggests that the D1-D2 interfering peptide specifically

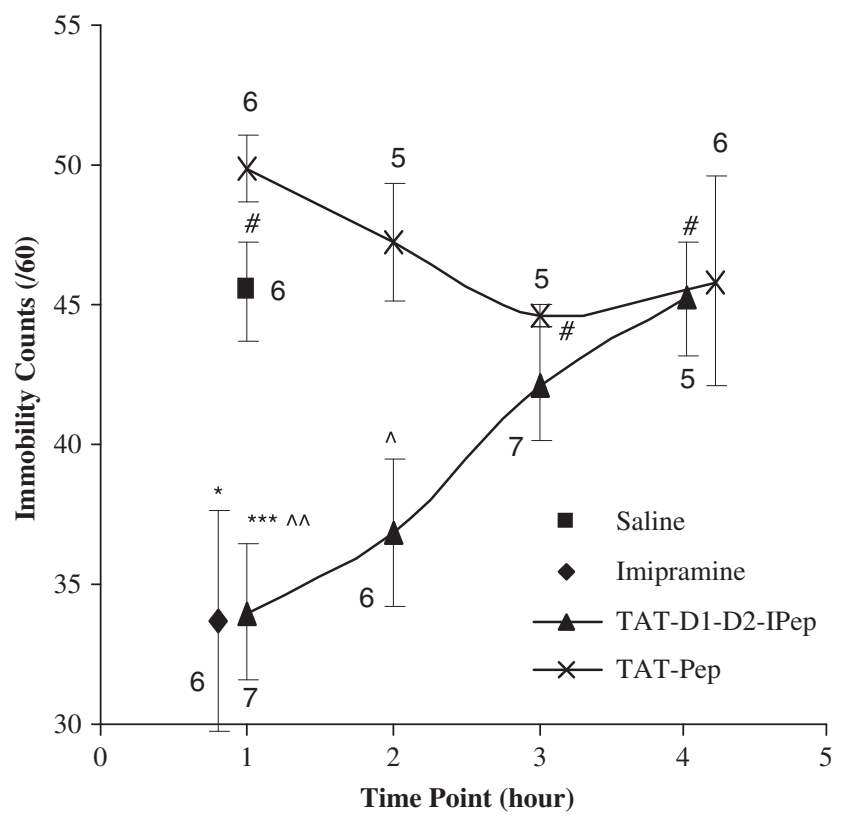

Figure 3 The DI-D2 interfering peptide has a behavioral antidepressant effect in the FST for $2 \mathrm{~h}$ after its administration. Immobility data from our original time point of I $\mathrm{h}$ after intranasal administration are included for the purposes of comparison. Imipramine and saline treatment groups received IP and IN injections, respectively, I $\mathrm{h}$ before the FST. Immobility behavioral data from each time point $(I, 2,3$, and $4 \mathrm{~h}$ ) were analyzed using one-way independent groups ANOVA followed by Newman-Keuls post hoc tests. *** $p<0.00$ I, $* p<0.05$ compared with saline; $\wedge \wedge p<0.01, \wedge p<0.05$ compared with TAT-Peptide; ${ }^{\#} p<0.05$ compared with imipramine. Numbers below data points represent number of animals per group. Error bars represent SEM.

disrupts the protein-protein interaction between D1 and D2 receptors.

\section{IN Administration of the D1-D2 Interfering Peptide Does Not Increase Locomotor Activity During a 30-Min Activity Test}

We examined the effect of the D1-D2 interfering peptide on locomotor activity to investigate whether the anti-immobility effect of the D1-D2 interfering peptide in the FST was due to its specific anti-depressant effects or to any resultant global increase in locomotor activity in the D1-D2 interfering peptide treatment group. To test this, we compared the amount of locomotor activity during a 30-min activity test in animals given IN injections of saline $(n=5)$, TAT-Pep (IN, $2 \mathrm{nmol} / \mathrm{g}, n=5$ ), TAT-D1-D2-IPep (IN, $2 \mathrm{nmol} / \mathrm{g}, \quad n=5$ ), or imipramine (IP, $15 \mathrm{mg} / \mathrm{kg}, n=5$ ). A one-way independent groups ANOVA of locomotor activity (as measured by Beam Breaks) revealed a significant difference among all four treatment groups $(n=5$ per group, $\mathrm{F}(3,16)=9.775 p<0.001$; Figure 5a). Animals in the D1-D2 interfering peptide, TAT-peptide, or imipramine treatment groups had significantly lower overall locomotor activity during the 30-min test than animals in the saline group ( $n=5$ per group, all groups compared with saline treatment). We also compared the time course of locomotor activity between these groups using a two-way independent 
a

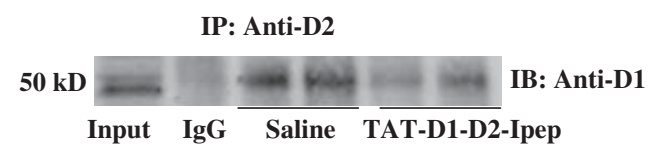

C

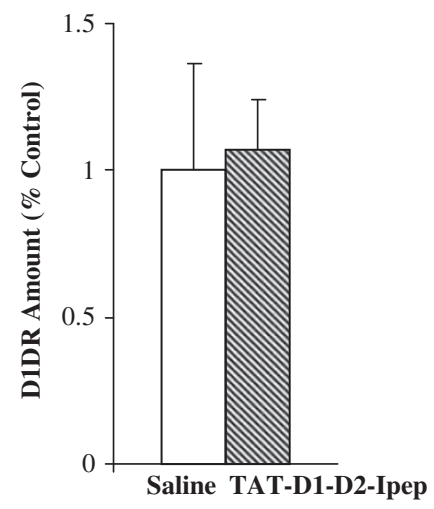

b

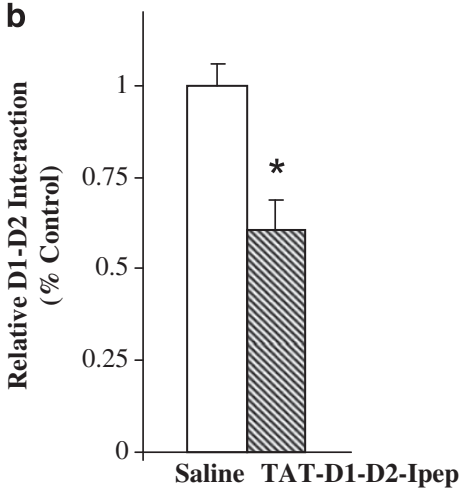

d

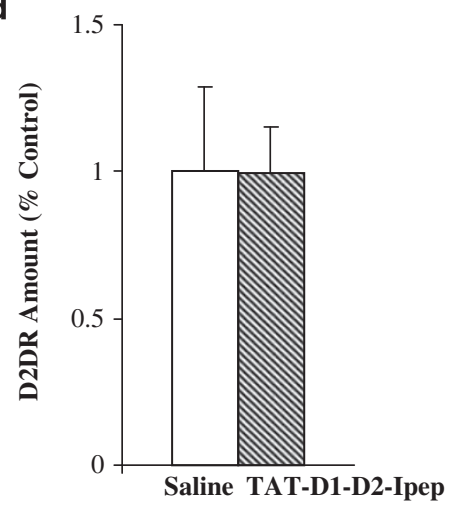

Figure 4 Intranasal administration of the DI-D2 interfering peptide disrupts the protein-protein interaction between DI and D2 receptors in the prefrontal cortex. (a) Representative immunoblot of anti-D2DR immunoprecipitated tissue from the PFC of rats that received intranasal injections of saline $(n=3)$ or TAT-DI-D2-Ipep (IN, $1.67 \mathrm{nmol} / \mathrm{g}, n=3)$. Input lane: $75 \mu \mathrm{g}$ solubilized PFC tissue, IgG: tissue incubated with nonspecific immunoglobulin antibody. (b) The interaction between DI and D2R is significantly reduced in the PFC of animals who received TAT-DI-D2-IPep. The interaction between $\mathrm{DI}$ and D2R was quantified by densitometry analysis of immunoblots. All samples were standardized to control (saline) samples and analyzed by two-tailed, unpaired Student's t-test $(n=3$ per group $=0.0$ l 8 ). (c) Quantitative densitometry analysis of immunoblot of DIR in PFC tissue from animals who received intranasal injections of TAT-DI-D2-IPep (IN, I.67 nmol/g, $n=3)$ or saline $(I N, n=3)$ revealed no significant differences between DI receptor expression $(n=3$ per group, $p>0.05)$. Error bars represent SEM. (d) Quantitative densitometry analysis of immunoblot of D2R in PFC tissue from animals who received intranasal injections of TAT-DI-D2-IPep (IN, $1.67 \mathrm{nmol} / \mathrm{g}, n=3)$ or saline $(\mathrm{IN}, n=3)$ revealed no significant differences between D2 receptor expression. Data were quantified using densitometry and analyzed using unpaired, two-tailed Student's t-test (* $p>0.05)$.

groups ANOVA, with treatment and time point as main factors. The analysis revealed a significant main effect of treatment groups $(\mathrm{F}(3,96)=20.24, p<0.001)$ and time point $(F(5,96)=30.66, p<0.001)$. The interaction between treatment group and time point was not significant $(F(15,96)=1.06, p>0.05$; Figure $5 b)$. From these results we can conclude that the D1-D2 interfering peptide is not increasing motor activity in a global manner, and thus is not acting as a stimulant in the FST.

\section{DISCUSSION}

Our study investigates whether peptide therapies designed to disrupt pathological interactions between two membrane receptors can be effectively delivered to the CNS in a noninvasive manner. We show that the D1-D2 interfering peptide, designed to disrupt the interaction between D1 and D2 dopamine receptors (Pei et al, 2010), has an antidepressant effect in the FST after IN delivery (Figure 2a). We demonstrate that the POD used in this study deposits a FLAG-tagged D1-D2 interfering peptide in the PFC in detectable amounts (Figure 1b). Administration of the D1D2 interfering peptide at doses of $>5.75 \mathrm{mg} / \mathrm{kg}(1.67 \mathrm{nmol} / \mathrm{g})$ has a significant anti-immobility effect that was comparable to that of imipramine in the FST, for up to $2 \mathrm{~h}$ after IN administration (Figures $2 \mathrm{~b}$ and 3 ). These findings demonstrate the efficacy of the IN pathway for peptide delivery to the CNS, expanded upon our laboratory's previous findings that the D1-D2 interfering peptide had an antidepressant effect in animal models of depression (Pei et al, 2010), and provide a solid basis for further development of this therapy as a noninvasive, clinically applicable treatment for MDD.

In animals that received POD injections of the D1-D2FLAG-tagged interfering peptide, we detected Cy-2 fluorescence conjugated to anti-FLAG antibodies both intracellularly and in extracellular areas of PFC slices (Figure 1a). We focused on investigating whether we could detect the D1-D2-FLAG peptide in the prefrontal cortex because in our laboratory's previous study, it only had an antidepressant effect when infused directly to the prefrontal cortex and not in other brain areas (Pei et al, 2010). Our results suggest that the PFC D1-D2 complex may be involved in the pathophysiology of depression. Thus, the PFC is the likely 

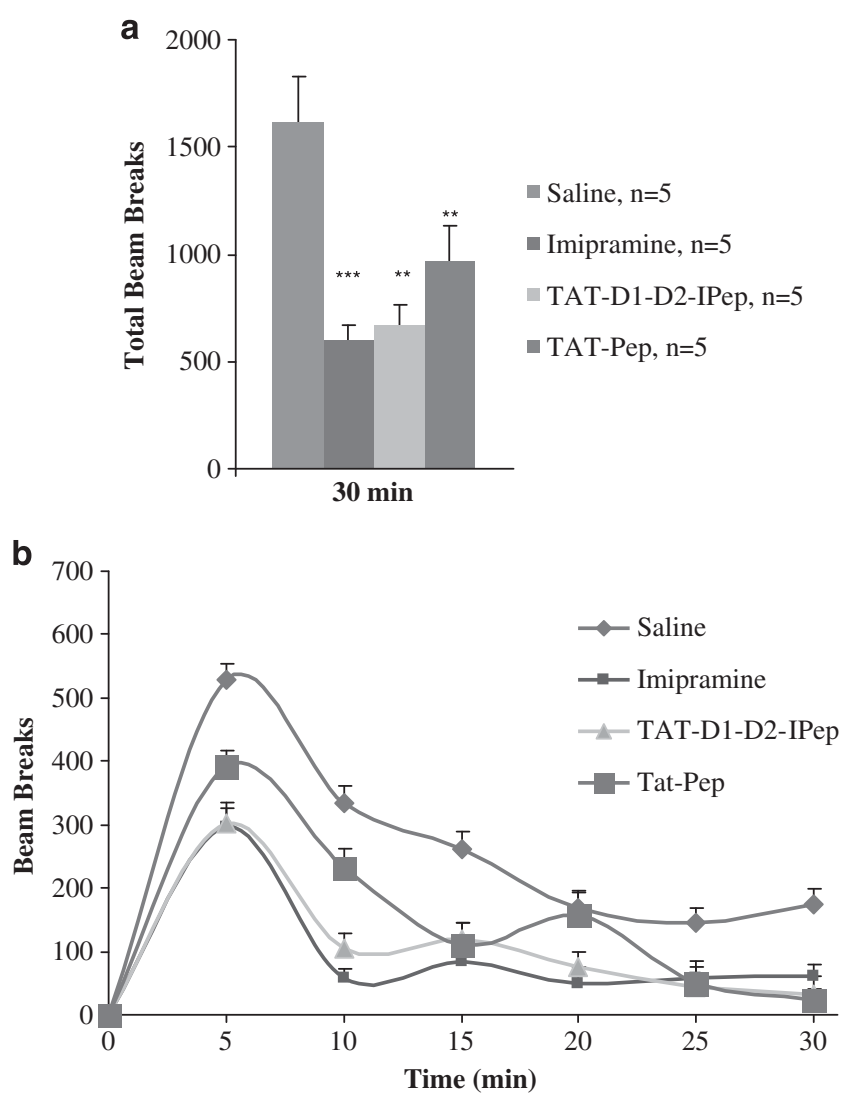

Figure 5 The DI-D2 interfering peptide does not increase locomotor activity during a 30-min open field test. (a) Total locomotor activity during a 30-min open field test. Locomotor activity was assessed during a 30-min open field test in a novel environment, and measured by the amount of movement around the testing chamber (Beam Breaks). Animals were given intranasal injections of saline, TAT-DI-D2-IPep, or TAT Pep $(2.0 \mathrm{nmol} / \mathrm{g})$ or IP injections of imipramine at 24, 19, and I $\mathrm{h}$ before the open field test. Locomotor activity data were analyzed by one-way independent groups ANOVA $(p<0.001)$ followed by post hoc Newman-Keuls multiple comparisons tests. ${ }^{*} * * * 0.001$, ${ }^{*} * p<0.01$, compared with saline. (b) The DI-D2 interfering peptide decreases overall locomotor activity but does not change the activity pattern during a 30-min open field test. Each Point on the test represents total number of beam breaks during the previous 5-min of the open field test. Data across treatment group and time points were analyzed using two-way independent groups ANOVA with time point and treatment group as independent factors. There was a significant main effect for time course $(p<0.00 \mathrm{I})$ and treatment group $(p<0.00 \mathrm{I})$ but no significant interaction between the two factors. Each group has $n=5$ animals. TAT-DI-D2-Ipep and TAT-Pep were given at an intranasal dose of $2.0 \mathrm{nmol} / \mathrm{g}$. Error bars represent SEM.

site of action for this interfering peptide to exert antidepressant-like effects.

One of the shortcomings of this study is that it was difficult to quantify the proportion of our original dose of D1-D2 interfering peptide that was effectively delivered into the neuronal cells of the PFC. However, we observed an antidepressant effect in the FST at an IN D1-D2 interfering peptide dose of $\geqslant 1.67 \mathrm{nmol} / \mathrm{g}, \sim 100$-fold larger than that given directly to the prefrontal cortex ( $5 \mathrm{nmol}$ per injection) in our previous study (Pei et al, 2010). We chose this starting dose based on previous studies of IN administration of small proteins such as NGF and insulin to the CNS

that indicated that the efficiency of delivery to the CNS is between 1 and 5\% (De Rosa et al, 2005; Dhuria et al, 2010; Francis et al, 2008; Marks et al, 2009). Although the proportion of the original IN dose of the D1-D2 interfering peptide present in the PFC after IN administration remains unknown, our results suggest that the efficiency of delivery to the CNS after IN administration using the POD device is at least $1-5 \%$. Another shortcoming of this study is that we used FST, an animal model for testing antidepressant-like effect. It is important to be aware that FST is not an animal model of depression.

Numerous previous studies regarding protein and peptide delivery to the CNS have indicated that proteins such as NGF (Chen et al, 1998; De Rosa et al, 2005), insulin (Francis et al, 2008; Renner et al, 2012; Shemesh et al, 2012), and IGF-1 (Fletcher et al, 2009; Liu et al, 2001; Thorne et al, 2004) preferentially enter the CNS through extracellular channels between the olfactory receptor neuron (ORN) axons and the olfactory ensheathing cells protecting them from the cribiform plate (Dhuria et al, 2010; Wu et al, 2008). As a result, it is probable that preferentially depositing substances onto the olfactory epithelium where ORN axons originate would increase the amount of D1-D2 interfering peptide delivered to the CNS while minimizing loss to the periphery. The POD is designed to preferentially deposit substances on the olfactory epithelium (Figure 1a) and, hypothetically, once there, substances are then transported to the CNS via extracellular pathways around the olfactory receptor neuron axons.

However, the mechanism by which the D1-D2 interfering peptide is transported to the CNS and PFC remains unknown. It is likely that the D1-D2 interfering peptide is being transported to the CNS via extracellular mechanisms, as the POD device preferentially deposits substances on the olfactory epithelium (Hoekman and Ho, 2011a,b), relatively short time is required for transport to the PFC (Figure 3), and previous studies demonstrate that peptides of this type are transported to the CNS via extracellular mechanisms (Baker and Spencer, 1986; Jansson and Bjork, 2002; Yang et al, 2013). A recent study by Yang et al (2013) showed that for a 22-amino acid, TAT-linked membrane-permeable peptide similar in size to the D1-D2 interfering peptide, an IN dose of only $7 \%$ that of the IV dose previously administered (Nijboer et al, 2008) was able to alleviate hypoxia-induced ischemic brain injury in a rat preclinical model. The authors also demonstrate that their TAT-linked peptide was detectable in the olfactory bulbs and anterior brain areas such as the PFC at only 10-30 min after delivery (Yang et al, 2013), a result that suggests that their peptide was being transported to the CNS via the extracellular pathways in the olfactory epithelium. Alternately, the peptide could be transported intracellularly by the olfactory receptor, a process that requires diffusion of the peptide into the ORNs and subsequent transport via axonal transport mechanisms to the axon terminals in the olfactory bulbs (Dhuria et al, 2010). This is unlikely, as previous studies have shown that intracellular transport of this type takes significantly longer than $1 \mathrm{~h}$ (Dhuria et al, 2010; Wu et al, 2008).

A third possibility is that the D1-D2 interfering peptide is absorbed into the systemic circulation through the nasal capillary bed, as the nasal cavity contains extensive 
capillaries and vascularization. After uptake into the bloodstream, the peptide would be transported throughout the body via systemic circulation, resulting in diffuse administration of the peptide and proportionally less of the IN dose transported to the CNS. Second, the peptide would need to cross the BBB, and, although peptides containing a membrane-permeable TAT sequence are permeable to the BBB (Schwarze et al, 1999), they require an extremely high systemic dose to enter the CNS in appreciable amounts, resulting in comparatively less of the starting dose reaching the CNS, and the PFC in particular. A number of studies from our laboratory support this, and suggest that TATlinked peptides need to be administered at a systemic dose of $3 \mathrm{nmol} / \mathrm{g}$ in order to be transported to, and have any pharmacological effect within the CNS. In the present study, the minimum effective dose we tested was $1.67 \mathrm{nmol} / \mathrm{g}$, $55 \%$ that of a typical systemic dose, implying that our IN delivery method has some specificity to the CNS. Although it is likely that after IN administration some of the D1-D2 peptide is taken up into the circulation, it is unlikely that this is the principle mechanism of transport to the CNS.

The D1-D2 interfering peptide has a significant antiimmobility effect in the FST at doses of $\geqslant 1.67 \mathrm{nmol} / \mathrm{g}$ $(5.75 \mathrm{mg} / \mathrm{kg}$; Figure 2). At these doses, the antidepressant effect of the D1-D2 interfering peptide in the FST is comparable to that of imipramine, a clinically effective tricyclic antidepressant. We further tested whether the behavioral response to the D1-D2 interfering peptide would differ at doses higher or lower than our original dose $(1.67 \mathrm{nmol} / \mathrm{g})$. We did not observe any appreciable increase or decrease in immobility behavior at D1-D2 interfering peptide doses of $>1.67 \mathrm{nmol} / \mathrm{g}$. Thus, there may be some threshold dose of the D1-D2 interfering peptide required to produce an anti-immobility effect in the FST, beyond which no additional antidepressant efficacy is conferred.

At the minimally effective IN dose, $1.67 \mathrm{nmol} / \mathrm{g}$, the D1-D2 interfering peptide has an anti-immobility effect in the FST for $2 \mathrm{~h}$, but not $3 \mathrm{~h}$, after IN administration. The absence of a behavioral effect at $3 \mathrm{~h}$ after IN administration is likely because of degradation of the D1-D2 interfering peptide as it is transported from the olfactory epithelium to the CNS, and within the CNS itself, as the mucous membranes in the nasal cavity and covering the olfactory epithelium contain proteases capable of degrading peptide bonds (Wu et al, 2008). Small peptides such as the D1-D2 interfering peptide disrupt the interaction between two proteins by competitively binding to the interacting regions, and, as such, must remain properly folded in order to effectively bind to these regions. The longer the D1-D2 interfering peptide is in the body, the more degradation occurs, resulting in loss of its pharmacological effects in the CNS. Strategies to improve the stability of the D1-D2 interfering peptide will be useful in increasing the duration of its pharmacological antidepressant effect.

Beyond the D1-D2 interfering peptide's ability to disrupt the interaction between D1 and D2 receptors in the PFC, the cellular and neurobiological mechanisms leading to its antidepressant efficacy remain unclear. Having said that, Pei et al (2010) document an increase in D1-D2 receptor interaction in the striatum of patients with MDD. This observation is correlative in nature and could be because of neurobiological changes from antidepressant treatments or a result of other confounding factors inherent in human studies. As such, no causal role for the D1R-D2R in the pathogenesis of MDD in the striatum has been shown. Although it is important to address, this lack of mechanistic insight is not uncommon for psychiatric medications, and should not necessarily hinder further development of this peptide as a novel antidepressant therapy. For example, beyond the acute increases in serotonin produced by SSRI-type antidepressants, the long-term neuronal changes that result in their ability to alleviate the symptoms of depression remain largely unknown (Belmaker and Agam, 2008; Mann, 2005).

One possible mechanic explanation for the antidepressant effects of the D1-D2 interfering peptide is that it causes an increase in the expression of BDNF in the prefrontal cortex and other brain areas. Unpublished data from our laboratory indicate that after ICV delivery of the D1-D2 interfering peptide to animals undergoing learned helplessness task, BDNF protein levels were elevated in PFC compared with animals that had received saline infusions (F Liu lab, unpublished data). Presumably, the D1-D2 interfering peptide has this effect because it blocks intracellular pathways that act to inhibit BDNF expression. Consistently, evidence from post-mortem human studies indicate that BDNF serum levels are decreased in the prefrontal cortex of patients diagnosed with MDD and committed suicide, and is increased in patients on long-term antidepressant treatment (Dwivedi et al, 2003; Karege et al, 2005; Pandey et al, 2008). However, a study from Hasbi et al (2009) suggests that D1-D2 receptor heterodimer activation could result in transcription of BDNF in cultured neurons derived from the ventral striatum. Taken together, it seems that the effect of BDNF levels on depression-like behaviors is region dependent (Autry and Monteggia, 2012) and the D1-D2 interfering peptide could have different downstream effect in different neuronal populations.

Interestingly, the D1-D2 interfering peptide, TAT peptide, and imipramine all significantly decreased locomotor activity during a 30-min test (Figure 5). When we examined locomotor activity in 5-min intervals, we found no significant interaction between treatment group and time (Figure 5b). This suggests that although the amount of locomotor activity was decreased in animals that received imipramine, TAT peptide, or D1-D2 interfering peptide as compared with the saline, the overall behavioral patterns did not change. Interestingly, in the study of Pei et al (2010), direct microinjections of the D1-D2 interfering peptide or the TAT peptide to the PFC, nucleus accumbens, or hippocampus did not significantly alter locomotor activity during a 30 -min open field test, whereas animals in the imipramine treatment group displayed decreased locomotor activity in the open field test, a result consistent with other studies of the sedative effects of imipramine in this test (Tucker and File, 1986). The difference in our results may be because of differences in testing methodology between this study and our laboratory's previous study (Pei et al, 2010). These include the method of administration (IN administration $v s$ direct microinjections), the fact that the IN doses of the D1-D2 interfering peptide and TAT peptide were much larger than those administered directly to the PFC, and the repeated use of isoflurane anesthetic in all groups. 
Our results demonstrate that the D1-D2 interfering peptide has an antidepressant effect in a pharmacologically valid preclinical test for antidepressant efficacy after IN delivery. Furthermore, our study has provided important, clinically relevant information vital to further development of the D1-D2 interfering peptide as a clinically viable treatment for MDD. At the same time, our results have raised a number of interesting questions about the antidepressant mechanism of the D1-D2 interfering peptide as well as the IN pathway used to deliver these peptides to the CNS. Further preclinical studies should be conducted to conclusively determine whether the D1-D2 interfering peptide can be tested as a novel antidepressant in the clinical setting.

\section{FUNDING AND DISCLOSURE}

The authors declare no conflict of interest.

\section{ACKNOWLEDGEMENTS}

We thank members in Dr Liu's lab (P Su, F Lee, S Li, D Zhai, and $\mathrm{T} \mathrm{Lu}$ ) for their technical support. We thank J Hokeman and Impel NeuroPharma for their advice regarding the use of the POD device. We thank Dr P Fletcher for his help with experimental design. $\mathrm{V}$ Brown conducted all experiments and co-wrote the manuscript. F Liu conceived of all experiments and co-wrote the manuscript. This work is supported by the Canadian Institutes for Health Research (CIHR; to FL).

\section{REFERENCES}

Autry AE, Monteggia LM (2012). Brain-derived neurotrophic factor and neuropsychiatric disorders. Pharmacol Rev 64: 238-258.

Baker H, Spencer RF (1986). Transneuronal transport of peroxidase-conjugated wheat germ agglutinin (WGA-HRP) from the olfactory epithelium to the brain of the adult rat. Exp Brain Res 63: 461-473.

Beaulieu JM, Gainetdinov RR (2011). The physiology, signaling, and pharmacology of dopamine receptors. Pharmacol Rev 63: 182-217.

Belmaker RH, Agam G (2008). Major depressive disorder. N Engl J Med 358: 55-68.

Chen XQ, Fawcett JR, Rahman YE, Ala TA, Frey IW (1998). Delivery of nerve growth factor to the brain via the olfactory pathway. J Alzheimers Dis 1: 35-44.

Cuijpers P (2011). The patient perspective in research on major depression. BMC Psychiatry 11: 89.

De Rosa R, Garcia AA, Braschi C, Capsoni S, Maffei L, Berardi N et al (2005). Intranasal administration of nerve growth factor (NGF) rescues recognition memory deficits in AD11 anti-NGF transgenic mice. Proc Natl Acad Sci USA 102: 3811-3816.

Dhuria SV, Hanson LR, Frey WH 2nd (2010). Intranasal delivery to the central nervous system: mechanisms and experimental considerations. J Pharm Sci 99: 1654-1673.

Dwivedi Y, Rizavi HS, Conley RR, Roberts RC, Tamminga CA, Pandey GN (2003). Altered gene expression of brain-derived neurotrophic factor and receptor tyrosine kinase B in postmortem brain of suicide subjects. Arch Gen Psychiatry 60: 804-815.

Fletcher L, Kohli S, Sprague SM, Scranton RA, Lipton SA, Parra A et al (2009). Intranasal delivery of erythropoietin plus insulinlike growth factor-I for acute neuroprotection in stroke. Laboratory investigation. J Neurosurg 111: 164-170.
Francis GJ, Martinez JA, Liu WQ, Xu K, Ayer A, Fine J et al (2008). Intranasal insulin prevents cognitive decline, cerebral atrophy and white matter changes in murine type I diabetic encephalopathy. Brain 131(Pt 12): 3311-3334.

Free RB, Hazelwood LA, Cabrera DM, Spalding HN, Namkung Y, Rankin ML et al (2007). D1 and D2 dopamine receptor expression is regulated by direct interaction with the chaperone protein calnexin. J Biol Chem 282: 21285-21300.

Hasbi A, O'Dowd BF, George SR (2011). Dopamine D1-D2 receptor heteromer signaling pathway in the brain: emerging physiological relevance. Mol Brain 4: 26.

Hoekman JD, Ho RJ (2011a). Effects of localized hydrophilic mannitol and hydrophobic nelfinavir administration targeted to olfactory epithelium on brain distribution. AAPS PharmSciTech 12: $534-543$.

Hoekman JD, Ho RJ (2011b). Enhanced analgesic responses after preferential delivery of morphine and fentanyl to the olfactory epithelium in rats. Anesth Analg 113: 641-651.

Hopp TP, Prickett KS, Price VL, Libby RT, March CJ, Cerretti DP et al (1988). A short polypeptide marker sequence useful for recombinant protein identification and purification. Nat Biotechnol 6: 1204-1210.

Jansson B, Bjork E (2002). Visualization of in vivo olfactory uptake and transfer using fluorescein dextran. J Drug Target 10: 379-386.

Karege F, Vaudan G, Schwald M, Perroud N, La Harpe R (2005). Neurotrophin levels in postmortem brains of suicide victims and the effects of antemortem diagnosis and psychotropic drugs. Brain Res Mol Brain Res 136: 29-37.

Lee FJ, Liu F (2004). Direct interactions between NMDA and D1 receptors: a tale of tails. Biochem Soc Trans 32(Pt 6): 1032-1036.

Liu F, Wan Q, Pristupa ZB, Yu XM, Wang YT, Niznik HB (2000). Direct protein-protein coupling enables cross-talk between dopamine D5 and gamma-aminobutyric acid A receptors. Nature 403: 274-280.

Liu XF, Fawcett JR, Thorne RG, Frey WH 2nd (2001). Non-invasive intranasal insulin-like growth factor-I reduces infarct volume and improves neurologic function in rats following middle cerebral artery occlusion. Neurosci Lett 308: 91-94.

Lucki I (1997). The forced swimming test as a model for core and component behavioral effects of antidepressant drugs. Behav Pharmacol 8: 523-532.

Maggio R, Aloisi G, Silvano E, Rossi M, Millan MJ (2009). Heterodimerization of dopamine receptors: new insights into functional and therapeutic significance. Parkinsonism Relat Disord 15(Suppl 4): S2-S7.

Mann JJ (2005). The medical management of depression. N Engl J Med 353: 1819-1834.

Marks DR, Tucker K, Cavallin MA, Mast TG, Fadool DA (2009). Awake intranasal insulin delivery modifies protein complexes and alters memory, anxiety, and olfactory behaviors. J Neurosci 29: 6734-6751.

Missale C, Fiorentini C, Collo G, Spano P (2010). The neurobiology of dopamine receptors: evolution from the dual concept to heterodimer complexes. J Recept Signal Transduct Res 30: 347-354.

Mojtabai R (2009). Unmet need for treatment of major depression in the United States. Psychiatr Serv 60: 297-305.

Nijboer CH, Heijnen CJ, Groenendaal F, May MJ, van Bel F, Kavelaars A (2008). Strong neuroprotection by inhibition of NFkappaB after neonatal hypoxia-ischemia involves apoptotic mechanisms but is independent of cytokines. Stroke 39: 2129-2137.

O'Dowd BF, Nguyen T, Ji X, George SR (2013). D5 dopamine receptor carboxyl tail involved in D5-D2 heteromer formation. Biochem Biophys Res Commun 431: 586-589.

Pandey GN, Ren X, Rizavi HS, Conley RR, Roberts RC, Dwivedi Y (2008). Brain-derived neurotrophic factor and tyrosine kinase B receptor signalling in post-mortem brain of teenage suicide victims. Int J Neuropsychopharmacol 11: 1047-1061. 
Papakostas GI (2009). Managing partial response or nonresponse: switching, augmentation, and combination strategies for major depressive disorder. J Clin Psychiatry 70(Suppl 6): 16-25.

Pei L, Lee FJ, Moszczynska A, Vukusic B, Liu F (2004). Regulation of dopamine D1 receptor function by physical interaction with the NMDA receptors. J Neurosci 24: 1149-1158.

Pei L, Li S, Wang M, Diwan M, Anisman H, Fletcher PJ et al (2010). Uncoupling the dopamine D1-D2 receptor complex exerts antidepressant-like effects. Nat Med 16: 1393-1395.

Perreault ML, Hasbi A, Alijaniaram M, Fan T, Varghese G, Fletcher PJ et al (2010). The dopamine D1-D2 receptor heteromer localizes in dynorphin/enkephalin neurons: increased high affinity state following amphetamine and in schizophrenia. J Biol Chem 285: 36625-36634.

Porsolt RD, Anton G, Blavet N, Jalfre M (1978). Behavioural despair in rats: a new model sensitive to antidepressant treatments. Eur J Pharmacol 47: 379-391.

Porsolt RD, Le Pichon M, Jalfre M (1977). Depression: a new animal model sensitive to antidepressant treatments. Nature 266: 730-732.

Renner DB, Svitak AL, Gallus NJ, Ericson ME, Frey WH 2nd, Hanson LR (2012). Intranasal delivery of insulin via the olfactory nerve pathway. J Pharm Pharmacol 64: 1709-1714.

Ridray S, Griffon N, Mignon V, Souil E, Carboni S, Diaz J et al (1998). Coexpression of dopamine D1 and D3 receptors in islands of Calleja and shell of nucleus accumbens of the rat: opposite and synergistic functional interactions. Eur J Neurosci 10: $1676-1686$.

Ross TM, Zuckermann RN, Reinhard C, Frey WH 2nd (2008). Intranasal administration delivers peptoids to the rat central nervous system. Neurosci Lett 439: 30-33.

Schwartz JC, Diaz J, Bordet R, Griffon N, Perachon S, Pilon C et al (1998). Functional implications of multiple dopamine receptor subtypes: the D1/D3 receptor coexistence. Brain Res Brain Res Rev 26: 236-242.
Schwarze SR, Ho A, Vocero-Akbani A, Dowdy SF (1999). In vivo protein transduction: delivery of a biologically active protein into the mouse. Science 285: 1569-1572.

Shemesh E, Rudich A, Harman-Boehm I, Cukierman-Yaffe T (2012). Effect of intranasal insulin on cognitive function: a systematic review. J Clin Endocrinol Metabol 97: 366-376.

So CH, Verma V, Alijaniaram M, Cheng R, Rashid AJ, O'Dowd BF et al (2009). Calcium signaling by dopamine D5 receptor and D5-D2 receptor hetero-oligomers occurs by a mechanism distinct from that for dopamine D1-D2 receptor heterooligomers. Mol Pharmacol 75: 843-854.

Thase ME, Friedman ES, Biggs MM, Wisniewski SR, Trivedi MH, Luther JF et al (2007). Cognitive therapy versus medication in augmentation and switch strategies as second-step treatments: a $\mathrm{STAR}^{\star} \mathrm{D}$ report. Am J Psychiatry 164: 739-752.

Thorne RG, Pronk GJ, Padmanabhan V, Frey WH 2nd (2004). Delivery of insulin-like growth factor-I to the rat brain and spinal cord along olfactory and trigeminal pathways following intranasal administration. Neuroscience 127: 481-496.

Tucker JC, File SE (1986). The effects of tricyclic and 'atypical' antidepressants on spontaneous locomotor activity in rodents. Neurosci Biobehav Rev 10: 115-121.

van Beljouw I, Verhaak P, Prins M, Cuijpers P, Penninx B, Bensing J (2010). Reasons and determinants for not receiving treatment for common mental disorders. Psychiatr Serv 61: 250-257.

Wong AH, Liu F (2012). Uncoupling the dopamine D1-D2 receptor complex: a novel target for antidepressant treatment. Clin Pharmacol Ther 91: 298-302.

Wu H, Hu K, Jiang X (2008). From nose to brain: understanding transport capacity and transport rate of drugs. Expert Opin Drug Deliv 5: 1159-1168.

Yang D, Sun YY, Lin X, Baumann JM, Dunn RS, Lindquist DM et al (2013). Intranasal delivery of cell-penetrating anti-NF-kappaB peptides (Tat-NBD) alleviates infection-sensitized hypoxicischemic brain injury. Exp Neurol 247: 447-455. 